\title{
Solubility of Anthracene in Binary Propyl Acetate + Alcohol Solvent Mixtures at 298.15 K
}

\author{
Lisset Alcazar, Ana Blanco, Ruth Cano, Leigh Fisher, Mason Nau, Lizette Sidransky, and William E. Acree, Jr.*
}

Department of Chemistry, P.O. Box 305070, University of North Texas, Denton, Texas 76203-5070

\begin{abstract}
Experimental solubilities are reported for anthracene in six binary propyl acetate + alcohol solvent mixtures at $298.15 \mathrm{~K}$. The alcohol solvents studied were 1-propanol, 1-butanol, 2-propanol, 2-butanol, 2-methyl-1propanol, and 3-methyl-1-butanol. Results of these measurements were used to test a mathematical representation based on the combined nearly ideal binary solvent (NIBS)/Redlich-Kister equation. For the six systems studied, the combined NIBS/Redlich-Kister equation was found to accurately describe the experimental data, with an average absolute deviation between measured and back-calculated values being approximately $\pm 0.8 \%$.
\end{abstract}

\section{Introduction}

Solubility plays an essential role in many manufacturing processes, especially in the purification of organic compounds through recrystallization and liquid extraction. Over the past 20 years, we have reported experimental solubility data for anthracene and pyrene dissolved in numerous binary solvents and have developed a fairly simple predictive method for estimating the solubility of crystalline organic compounds in ternary mixtures ${ }^{1-4}$

$$
\begin{array}{r}
\ln x_{\mathrm{A}}{ }^{\mathrm{sat}}=x_{\mathrm{B}}{ }^{\mathrm{o}} \ln \left(x_{\mathrm{A}}{ }^{\mathrm{sat}}\right)_{\mathrm{B}}+x_{\mathrm{C}}{ }^{\mathrm{o}} \ln \left(x_{\mathrm{A}}{ }^{\mathrm{sat}}\right)_{\mathrm{C}}+x_{\mathrm{D}}{ }^{\mathrm{o}} \ln \left(x_{\mathrm{A}}{ }^{\mathrm{sat}}\right)_{\mathrm{D}}+ \\
x_{\mathrm{B}}{ }^{\mathrm{o}} x_{\mathrm{C}}{ }_{i=0}^{\mathrm{o}} \sum_{i=0}^{r} S_{\mathrm{BC}, i}\left(x_{\mathrm{B}}{ }^{\mathrm{o}}-x_{\mathrm{C}}{ }^{\mathrm{o}}{ }^{i}+x_{\mathrm{B}}{ }^{\mathrm{o}} x_{\mathrm{D}}{ }^{\mathrm{o}} \sum_{j=0}^{s} S_{\mathrm{BD}, j}\left(x_{\mathrm{B}}{ }^{\mathrm{o}}-x_{\mathrm{D}}{ }^{\mathrm{o}}\right)^{j}+\right. \\
x_{\mathrm{C}}{ }^{\mathrm{o}} x_{\mathrm{D}}{ }^{\mathrm{o}} \sum_{k=0}^{t} S_{\mathrm{CD}, k}\left(x_{\mathrm{C}}{ }^{\mathrm{o}}-x_{\mathrm{D}}{ }^{\mathrm{o}}\right)^{k}
\end{array}
$$

and higher-order multicomponent solvent mixtures ${ }^{5}$

$$
\ln x_{\mathrm{A}}^{\mathrm{sat}}=\sum_{I} x_{I}^{\mathrm{o}} \ln \left(x_{\mathrm{A}}^{\mathrm{sat}}\right)_{I}+\sum_{I} \sum_{J>I}\left(x_{I}^{\mathrm{o}} x_{J}^{\mathrm{o}} \sum_{i=0}^{r} S_{I J, i}\left(x_{I}^{\mathrm{o}}-x_{J}^{\mathrm{o}}\right)^{i}\right)
$$

based on the extended form of the combined nearly ideal binary solvent (NIBS)/Redlich-Kister solution model. Predictions are based on the measured solubility data in all of the contributing sub-binary solvent mixtures. In eqs 1 and $2, x_{I}^{\circ}$ refers to the initial mole fraction-solvent composition of component $i$ calculated as if the solute were not present, and $\left(x_{\mathrm{A}}\right)_{I}$ denotes the measured solute solubility in pure solvent $i$. The various $S_{I J, i}$ parameters can be evaluated with a least-squares regression analysis.

In the present study, we extend our solubility measurements to binary solvent mixtures containing propyl acetate and an alcohol cosolvent. Experimental solubilities are reported for anthracene dissolved in six binary mixtures containing propyl acetate with 1-propanol, 2-propanol, 1-butanol, 2-butanol, 2-methyl-1-butanol, and 3-methyl-1-butanol at 298.15 K. Results

\footnotetext{
* To whom correspondence should be addressed. E-mail: acree@unt.edu.
}

of these measurements are used to calculate the $S_{I J, i}$ curve-fit coefficients. To date, the solubility behavior of anthracene in binary propyl acetate + alcohol solvent mixtures has not been studied.

\section{Experimental Methods}

Anthracene (Aldrich, 99+\%) was recrystallized several times from 2-propanone to yield a purified sample having a melting point temperature of $T / \mathrm{K}=489$. Propyl acetate (Aldrich, 99 $\%$, anhydrous), 1-propanol (Aldrich, 99+ \%, anhydrous), 2-propanol (Aldrich, 99+ \%, anhydrous), 1-butanol (Aldrich, $99+\%$, anhydrous), 2-butanol (Aldrich, 99+ \%, anydrous), 2-methyl-1-propanol (Aldrich, 99+\%, anhydrous), and 3-methyl-1-butanol (Aldrich, $99 \%$, anhydrous) were stored over molecular sieves and distilled shortly before use. Gas chromatographic analysis showed the solvent mole fraction purities to be $99.7 \%$ or better. Binary solvent mixtures were prepared by mass so that composition could be calculated to 0.0001 mole fraction.

Excess solute and solvent were placed in amber glass bottles and allowed to equilibrate in a constant-temperature water bath at $T / \mathrm{K}=298.15 \pm 0.05$ for at least three days (often longer) with periodic shaking to facilitate dissolution of the solid. Attainment of equilibrium was verified by repetitive measurements after a minimum of three additional days and by approaching equilibrium from supersaturation by pre-equilibrating the solutions at a slightly higher temperature. Aliquots of saturated anthracene solutions were transferred through a coarse filter into a tared volumetric flask to determine the amount of sample and then diluted quantitatively with methanol for spectrophotometric analysis at $356 \mathrm{~nm}$ on a Bausch and Lomb Spectronic 2000. Concentrations of the dilute solutions were determined from a Beer-Lambert law absorbance versus concentration working curve. Molar absorptivities of the nine standard solutions varied systematically with molar concentration and ranged from $\epsilon /\left(\mathrm{L} \cdot \mathrm{mol}^{-1} \cdot \mathrm{cm}^{-1}\right)=7450$ to $\epsilon /\left(\mathrm{L} \cdot \mathrm{mol}^{-1} \cdot \mathrm{cm}^{-1}\right)=$ 7150 for anthracene compositions ranging from $C /\left(\mathrm{mol} \cdot \mathrm{L}^{-1}\right)$ $=6.75 \cdot 10^{-5}$ to $C /\left(\mathrm{mol} \cdot \mathrm{L}^{-1}\right)=2.25 \cdot 10^{-4}$. Identical molar absorptivities were obtained for select anthracene standard solutions that contained volume fractions up to $5 \%$ of the 
Table 1. Experimental Mole Fraction Solubilities, $x_{\mathrm{A}}{ }^{\text {sat }}$, of Anthracene in Binary Propyl Acetate (B) + Alcohol (C) Solvent Mixtures at $298.15 \mathrm{~K}$

\begin{tabular}{|c|c|c|c|}
\hline$x_{\mathrm{B}}{ }^{\mathrm{o}}$ & $x_{\mathrm{A}}{ }^{\text {sat }}$ & $x_{\mathrm{B}}{ }^{\mathrm{o}}$ & $x_{\mathrm{A}}{ }^{\text {sat }}$ \\
\hline \multicolumn{4}{|c|}{ Propyl Acetate (B) + 1-Propanol (C) } \\
\hline 0.0000 & 0.000591 & 0.5377 & 0.00309 \\
\hline 0.0872 & 0.000917 & 0.7587 & 0.00438 \\
\hline 0.1634 & 0.001211 & 0.8699 & 0.00504 \\
\hline 0.3403 & 0.00205 & 1.0000 & 0.00588 \\
\hline 0.4416 & 0.00252 & & \\
\hline \multicolumn{4}{|c|}{ Propyl Acetate (B) + 2-Propanol (C) } \\
\hline 0.0000 & 0.000411 & 0.5016 & 0.00280 \\
\hline 0.0714 & 0.000672 & 0.7254 & 0.00410 \\
\hline 0.1352 & 0.000931 & 0.8374 & 0.00479 \\
\hline 0.3510 & 0.001973 & & \\
\hline 0.4018 & 0.00225 & & \\
\hline \multicolumn{4}{|c|}{ Propyl Acetate (B) + 1-Butanol (C) } \\
\hline 0.0000 & 0.000801 & 0.5377 & 0.00352 \\
\hline 0.0872 & 0.001189 & 0.7587 & 0.00478 \\
\hline 0.1634 & 0.001548 & 0.8699 & 0.00538 \\
\hline 0.3403 & 0.00241 & & \\
\hline 0.4416 & 0.00297 & & \\
\hline \multicolumn{4}{|c|}{ Propyl Acetate (B) + 2-Butanol (C) } \\
\hline 0.0000 & 0.000585 & 0.5447 & 0.00335 \\
\hline 0.0842 & 0.000946 & 0.7581 & 0.00464 \\
\hline 0.1636 & 0.001311 & 0.8652 & 0.00523 \\
\hline 0.3427 & 0.00221 & & \\
\hline 0.4310 & 0.00269 & & \\
\hline \multicolumn{4}{|c|}{ Propyl Acetate (B) + 2-Methyl-1-propanol (C) } \\
\hline 0.0000 & 0.000470 & 0.5508 & 0.00309 \\
\hline 0.0853 & 0.000791 & 0.7562 & 0.00441 \\
\hline 0.1755 & 0.001185 & 0.8650 & 0.00513 \\
\hline 0.3477 & 0.001987 & & \\
\hline 0.4462 & 0.00248 & & \\
\hline \multicolumn{4}{|c|}{ Propyl Acetate (B) + 3-Methyl-1-butanol (C) } \\
\hline 0.0000 & 0.000727 & 0.5842 & 0.00354 \\
\hline 0.0890 & 0.001085 & 0.7905 & 0.00478 \\
\hline 0.1932 & 0.001552 & 0.8877 & 0.00532 \\
\hline 0.3811 & 0.00242 & & \\
\hline 0.4848 & 0.00294 & & \\
\hline
\end{tabular}

neat propyl acetate and alcohol cosolvents. Experimental molar concentrations were converted to mass fraction solubility by multiplying by the molar mass of anthracene, volume(s) of the volumetric flask(s) used, and any dilutions required to place the measured absorbances on the Beer-Lambert law absorbance versus concentration working curve and then dividing by the mass of the saturated solution analyzed. Mole fraction solubilities were computed from mass fraction solubility using the binary solvent initial mole fraction compositions and molar masses of the solute and both cosolvents.

Experimental anthracene solubilities in the six binary propyl acetate + alcohol mixtures studied are listed in Table 1 . Numerical values represent the average of between four and eight independent determinations, with the measured mole fraction solubilities being reproducible to within $\pm 1.5 \%$. Calculated standard deviations ranged from $\sigma_{n-1}=0.000004$ to $\sigma_{n-1}=0.000038$ mole fraction. The larger standard deviations were observed for the solutions having the larger mole fraction solubilities. Here, an extra dilution was required to get the measured absorbance to fall on the working curve established by the standard solutions.

\section{Results and Discussion}

For a binary solvent mixture, eq 2 takes the mathematical form of
Table 2. Mathematical Representation of Anthracene Solubilities in Several Binary Propyl Acetate (B) + Alcohol (C) Solvent Mixtures Based on eq (3)

\begin{tabular}{lrc}
\hline solvent $(\mathrm{B})+$ solvent $(\mathrm{C})$ & \multicolumn{1}{c}{$S_{i}^{a}$} & $100 \cdot \mathrm{dev}^{b}$ \\
\hline propyl acetate + 1-propanol & 1.707 & 0.9 \\
& -0.948 & \\
& 0.604 & \\
propyl acetate + 2-propanol & 2.274 & 1.0 \\
& -1.566 & \\
propyl acetate + 1-butanol & 1.132 & \\
& 1.656 & 0.5 \\
propyl acetate + 2-butanol & -0.706 & \\
& 0.729 & \\
& 1.994 & 0.9 \\
propyl acetate + 2-methyl-1-propanol & -1.118 & \\
& 1.026 & \\
& 2.048 & 0.7 \\
propyl acetate + 3-methyl-1-butanol & -1.218 & \\
& 1.187 & \\
& 1.544 & 0.6 \\
& -0.745 &
\end{tabular}

${ }^{a}$ Curve-fit coefficients are ordered as $S_{0}, S_{1}$, and $S_{2} \cdot{ }^{b}$ Deviation $=$ $\sum \mathrm{I}\left[\left(x_{\mathrm{A}}{ }^{\text {sat }}\right)^{\text {calcd }}-\left(x_{\mathrm{A}}{ }^{\text {sat }}\right)^{\text {exptl }}\right] /\left(x_{\mathrm{A}}{ }^{\text {sat }}\right)^{\text {exptl }} / / N$.

$$
\begin{aligned}
\ln x_{\mathrm{A}}{ }^{\mathrm{sat}}=x_{\mathrm{B}}{ }^{\mathrm{o}} \ln \left(x_{\mathrm{A}}{ }^{\mathrm{sat}}\right)_{\mathrm{B}}+x_{\mathrm{C}}{ }^{\mathrm{o}} \ln \left(x_{\mathrm{A}}{ }^{\mathrm{sat}}\right)_{\mathrm{C}}+ \\
x_{\mathrm{B}}{ }^{\mathrm{o}} x_{\mathrm{C}}{ }^{\mathrm{o}} \sum_{i=0}^{r} S_{\mathrm{BC}, i}\left(x_{\mathrm{A}}{ }^{\mathrm{sat}}-x_{\mathrm{C}}{ }^{\mathrm{o}}\right)^{i}
\end{aligned}
$$

The ability of eq 3 to represent mathematically the experimental solubility of anthracene in the six binary propyl acetate + alcohol solvent mixtures is summarized in Table 2 in the form of the "curve-fit" parameters and the average absolute percent deviations in back-calculated solubilities. The parameters $S$ were computed from a polynomial leastsquares analysis of

$$
\begin{array}{r}
{\left[\ln x_{\mathrm{A}}{ }^{\mathrm{sat}}-x_{\mathrm{B}}{ }^{\mathrm{o}} \ln \left(x_{\mathrm{A}}{ }^{\mathrm{sat}}\right)_{\mathrm{B}}-x_{\mathrm{C}}{ }^{\mathrm{o}} \ln \left(x_{\mathrm{A}}{ }^{\mathrm{sat}}\right)_{\mathrm{C}}\right] /\left(x_{\mathrm{B}}{ }^{\mathrm{o}} x_{\mathrm{C}}{ }^{\mathrm{o}}\right)=S_{0}+} \\
S_{1}\left(x_{\mathrm{B}}{ }^{\mathrm{o}}-x_{\mathrm{C}}{ }^{\mathrm{o}}\right)+S_{2}\left(x_{\mathrm{B}}{ }^{\mathrm{o}}-x_{\mathrm{C}}{ }^{\mathrm{o}}\right)^{2}
\end{array}
$$

using the commercial software TableCurve (Janel Scientific, Corte Madera, California). Careful examination of Table 2 reveals that eq 3 does provide an accurate mathematical representation for how the solubility of anthracene varies with solvent composition. The overall average absolute deviation between back-calculated and experimental values is $\pm 0.8 \%$, which is less than the uncertainty associated with the measured mole fraction solubilities. The calculated curve-fit coefficients, when combined with our previously reported values for anthracene dissolved in binary alcohol + alcohol $^{6,7}$ solvent mixtures, allow one to estimate the solubility of anthracene in ternary propyl acetate + alcohol + alcohol solvent mixtures containing the alcohol cosolvents of 1-propanol, 2-propanol, 1-butanol, 2-butanol, 2-methyl-1-propanol, and 3-methyl-1butanol.

\section{Literature Cited}

(1) Deng, T.; Acree, W. E., Jr. Solubility of anthracene in ternary propanol $+2,2,4$-trimethylpentane + cyclohexane and butanol $+2,2,4$-trimethylpentane + cyclohexane solvent mixtures. J. Chem. Eng. Data 1998, 43, 1059-1061.

(2) Deng, T.; Hernández, C. E.; Roy, L. E.; Acree, W. E., Jr. Solubility of anthracene in (ternary propanol + heptane + cyclohexane) and (butanol + heptane + cyclohexane) solvent mixtures. J. Chem. Thermodyn. 1999, $31,205-210$.

(3) Deng, T.; Acree, W. E., Jr. Solubility of anthracene in ternary propanol + butanol + cyclohexane solvent mixtures. J. Chem. Eng. Data 1998, $43,1062-1064$. 
(4) Debase, E. M.; Acree, W. E., Jr. Solubility of pyrene in ternary propanol + butanol + cyclohexane solvent mixtures at 299.15 K. J. Chem. Eng. Data 2001, 46, 991-993.

(5) Deng, T.; Horiuchi, S.; De Fina, K. M.; Hernández, C. E.; Acree, W. E., Jr. Solubility of anthracene in multicomponent solvent mixtures containing propanol, butanol and alkanes. J. Chem. Eng. Data 1999, 44, 798-802.

(6) Acree, W. E., Jr.; Zvaigzne, A. I. Thermochemical investigations of hydrogen-bonded solutions. Part 5. Development of predictive equations for the solubility of anthracene in binary alcohol + alcohol mixtures based upon mobile order theory. Fluid Phase Equilib. 1994, 99, 167183.
(7) Zvaigzne, A. I.; Acree, W. E., Jr. Solubility of anthracene in binary alcohol + 2-methyl-1-propanol and alcohol +3 -methyl-1-butanol solvent mixtures. J. Chem. Eng. Data 1995, 40, 917-919.

Received for review September 03, 2007. Accepted October 08, 2007. The authors thank the University of North Texas Research Council for partial support of this work. Lisset Alcazar, Ana Blanco, Ruth Cano, Leigh Fisher, Mason Nau, and Lizette Sidransky thank the U.S. Department of Education for the support provided to them under the Upward Bound Math and Science Program.

JE700502X 